Abstract 246 Table 1 Describes change in key health measures. $40 \%$ of CYP and $43 \%$ of parents reported a deterioration in mental health but only 6 families accessed emotional support groups

\begin{tabular}{lllll}
\hline & Better & Same & Worse & n/a \\
\hline CYP Mental Health & $13 \%$ & $47 \%$ & $40 \%$ & \\
Parent Mental Health & $8 \%$ & $49 \%$ & $43 \%$ & \\
CYP Physical Health & $19 \%$ & $48 \%$ & $33 \%$ & \\
Parent Physical Health & $9 \%$ & $59 \%$ & $32 \%$ & \\
CYP exercise & $8 \%$ & $33 \%$ & $56 \%$ & $3 \%$ \\
CYP sleep & $7 \%$ & $50 \%$ & $43 \%$ & \\
Finance & $4 \%$ & $55 \%$ & $41 \%$ & \\
Housing & $4 \%$ & $83 \%$ & $13 \%$ & \\
Employment & $0 \%$ & $63 \%$ & $37 \%$ & \\
\hline
\end{tabular}

social and health needs of this population and shape our future service.

Methods A questionnaire was designed and piloted with two parents. The sample was non-random/convenience sampling taken from a contact list of CYP with SEND who were shielding or considered vulnerable. Participants were informed this was part of a service evaluation and consented to be interviewed. A total of 75 telephone interviews were conducted. The council completed 46 questionnaires and in October 2020 the medical team attempted contact with a further 94 families. 46 families answered the telephone and 29 agreed to complete the questionnaire. Ethics was not sought as it was a health service evaluation.

Results The participants children were between 0-19 years with the majority $(40 \%)$ between 5-9 years. $89 \%$ of the sample was BAME. 55\% of the school-age children were eligible for a school place during lockdown however only 21\% of these opted to attend school. Parents cited reasons for not attending school as shielding, fear of COVID-19 and lack of equipment.

The most common services accessed were primary care, specialist services (eg child development centre) and food banks. Of the 27 families describing worse employment situation, only 1 accessed the jobcentre. Of the 31 families with worse financial situation 15 accessed the food bank and 1 accessed the Citizens' Advice Bureau.

Conclusions Further exploration is required with validated measures to understand the impact of the pandemic and of associated interventions (eg lockdown) on health and mental health in particular. Our survey shows that emotional and financial services were not widely used despite the difficulties many faced. Signposting families to these services is being prioritised in our clinical interactions and through development of a social prescribing model. More in-depth qualitative research is planned on this population exploring the interplay between social determinants and COVID in CYP with SEND and their families.

\section{VALIDITY OF AN IVH AND PVL PREVENTION BUNDLE INTERVENTIONS IN A CENTRE WITH A VERY LOW INCIDENCE OF SEVERE IVH: A CASE-CONTROL STUDY}

Mavis Xuanru Tan, Abdul Alim Abdul Haium, Nirmal Kavalloor Visruthan, Victor Samuel Rajadurai, Thowfique Ibrahim. Singapore
Background Severe intraventricular haemorrhage (IVH, Papile's Grades 3 and 4) can cause significant neurodevelopmental impairment in $43 \%$ of affected infants.

Objectives The objectives of this study were to study the risk factors for severe IVH and evaluate the validity of an IVH prevention bundle in VLBW infants in a tertiary care centre with very low incidence of severe IVH.

Methods All VLBW infants born between January 2013 to December 2018 were included in the study. Data was collected from a prospectively maintained database and retrospective chart review. For each severe IVH infant (study group), 2 IVH-free infants were identified as control and were closely matched for birth weight $( \pm 100 \mathrm{~g})$ and gestational age $( \pm 1$ week). Eighteen risk factors studied under severe IVH review document and six risk factors explored under interventions were combined, edited for overlaps or repeats and compared between the two groups. Bundle of interventions to reduce severe IVH included 1) Neutral head position and developmental care (2012); 2) Optimising the time of administration of antenatal steroid to mothers at risk of preterm birth at $\leq 24$ weeks gestation by administering the drug at least 48 hours before the delivery (2014); 3) Antenatal magnesium sulphate administration for neuroprotection to mothers in preterm labour with foetus of $\leq 32$ weeks gestation (2015); 4) Protocol for prevention of hypothermia in newborn infants (2016); 5) Patent ductus arteriosus (PDA) management protocol featuring early selective treatment of high-risk infants (2016); and 6) Prophylactic indomethacin for IVH prevention guideline (2016, modified in 2017). Univariate and multivariate analysis were conducted.

The bundle was defined as valid, if there was a statistically significant difference in incidence of corresponding risk factors between the 2 groups and $\geq 50 \%$ reduction in incidence of severe IVH in the post-intervention period. The stratified yearly incidences of IVH were captured.

Results 1261 VLBW infants were born during the study period and $48(3.8 \%)$ infants developed severe IVH with 96 matched infants identified as controls. Baseline maternal and infant characteristics were comparable. The incidence of severe IVH was $6.2 \%$ and $1.9 \%$ in the 2012 and 2018 cohorts respectively. On univariate analysis, maternal tocolytic therapy, difficult resuscitation at birth, difficult procedures, hypercapnia, high-frequency oscillatory ventilation and, multiple inotropes for hypotension during the first week of life were found to be statistically significant. Multivariate analysis revealed tocolytic therapy (OR $0.26,95 \% \mathrm{CI}, 0.10-0.66$ ) to be an independent protective factor whereas delivery room intubation (OR 8.77, 95\% CI, 1.10 - 70.06) and multiple inotropes (OR 2.32, 95\% CI, 1.44 - 3.76) were significant risk factors for severe IVH.

Conclusions The IVH prevention bundle interventions were found to be valid and the bundle model can be replicated to reduce the overall incidence of severe IVH in VLBW infants to less than $2 \%$.

\section{DOES THE 'CRIB II' SCORING SYSTEM SCORE WELL IN MORTALITY RISK PREDICTION IN INDIA?}

Amruta Phatak, Abhimanyu Niswade, Rajkumar Meshram, Swapnil Wathore. India

10.1136/bmjpo-2021-RCPCH.140

Background Introduction: 
Clinical Risk Index for Babies score II (CRIB II score) is a five-items scoring system to predict initial risk of mortality among neonates less than 32 weeks of gestation. Thus, making it an easy, quick and economical tool for early detection of preterm neonates' mortality risk in resource limited busy centres in India.

Objectives Objective:

To validate the efficacy of Clinical Risk Index for Babies score II in predicting pre-discharge neonatal mortality in early preterm neonates needing intensive care in a high resource tertiary care centre providing referral services in central India.

Methods Methods:

In this prospective observational study, after taking informed consent of parents and ethical committee clearance, the CRIB-II scores, which include birthweight, gestational age, sex, body temperature, and base excess, were recorded within the first hour of admission for 140 neonates of gestational age ranging between 28-31 weeks and birth weight ranging from $1000 \mathrm{~g}$ to $2499 \mathrm{~g}$; of both sexes born in a tertiary care institute of central India and admitted to its neonatal intensive care unit (NICU). Babies of gestation $<28$ weeks and $>31$ completed weeks, birth weight $<1000$ g, having lethal congenital malformations, delivery room deaths and those admitted after $1 \mathrm{hr}$ of birth were excluded. Outcome measure was in hospital death or discharge. The sensitivity and specificity of CRIB-II scores and its cut off point to predict mortality were examined using Receiver Operating Characteristic curves (ROC) with area under curve (AUC) indicating predictive accuracy. Its association with mortality was determined by Cox Regression Hazard analysis. Clinical parameters were compared between Non-survivors and survivors by performing independent t-test. A $\mathrm{p}<0.05$ was considered as statistically significant.

Results Results:

Male: female ratio was 0.92:1. The mean Gestational age was $30.27 \pm 0.89$ weeks, mean birth weight being 1599.75 \pm 282.35 g. CRIB II score ranged from 1-19 with a mean of $13.16 \pm 25.56$ among non survivors and mean of $5.66 \pm$ 2.24 among the survivors ( $\mathrm{p}$ value $=<0.0001$ ). The total mortality in the study was $47.1 \%(66 / 140)$. There was a progressive increase in mortality with increasing CRIB II score $(\mathrm{p}=0.001)$ and increase in survival with increasing birth weight, gestational age, body temperature and hospital stay ( $\mathrm{p}$ $<0.0001$ for each variable). CRIB II score $\geq 9$ cut off was found to be significantly associated with neonatal mortality with sensitivity, predictive value and specificity of $95.65 \%$, $95.65 \%$ and $95.77 \%$ respectively $(\mathrm{p}<0.001 ; 95 \%$ CI 0.98 (0.96-1.00); hazard ratio $=1.38)$. The ROC curve for CRIB II score was suggestive of AUC of 0.9868 ie $98 \%$ predictive accuracy.

Conclusions Conclusion:

The present study shows that the CRIB II score is a useful and reliable tool to prioritize the interventions in NICUs and will help to reduce the neonatal mortality rate and improve preterm neonates' survival in India if used appropriately in tertiary care centres.

\section{NEONATAL ECTOPIC ATRIAL TACHYCARDIA- MIND THE GAP (PR INTERVAL)!}

Ahmed Kamal, Pramod Nair. UK

10.1136/bmjpo-2021-RCPCH. 141
Background Whereas SVTs are well recognised in neonates with early diagnosis and management occasionally ectopic atrial tachycardia in neonates do happen infrequently and could be mis diagnosed as sinus tachycardia particularly if in the slower range

Objectives The tachycardia in these instances often tend to be incessant and can lead to tachycardia induced cardiomyopathy if the rate remains uncontrolled. The ECG changes are subtle and needs a trained eye to pick up this diagnosis.

Methods We present a case of a male infant who presented acutely to the hospital at age of 19 days with blood in the stool. He was born at term baby with mild intra uterine growth retardation but otherwise well at birth. He was noted to have hypospadias and was diagnosed as a case of cow's milk protein intolerance. During the admission he was incidentally noted to be tachycardic with a heart rate consistently between 180 and 200 beats per minute while otherwise being well and afebrile. His ECG showed narrow complex tachycardia at a rate between 180 to 200 beats per minute. Blood tests were overall satisfactory with an elevated troponin level and negative viral serology studies. An echocardiography done suggested mild left atrial and left ventricular dilatation with mild impairment of function. The case was discussed with the tertiary Cardiac team who felt this was more likely to be a sinus tachycardia and the patient was subsequently discharged. At age of 6 weeks, the child was seen in the outreach cardiac clinic. The ECGs (Current and previous were reviewed). It was noted that $\mathrm{P}$ waves were normal axis but negative in aVL, the PR interval was disproportionately long for the tachycardia. This suggested atrial tachycardia which was persistent. An echocardiogram showed a structurally normal heart with mild left heart volume overload and mildly impaired left ventricular function. The child was stuck in a slow atrial tachycardia rhythm which contributed towards the impaired function. Propranolol was started at a dose of $1 \mathrm{mg} / \mathrm{kg}$ TDS.

Results At further follow up at 7 months, the child was thriving and well, echocardiography showed that the cardiac dimensions were normal and the function had improved. ECGs and ambulatory monitoring showed normal rates and rhythms. The beta blockers were continued with a plan to wean in future.

Conclusions Ectopic atrial tachycardia (EAT) in a neonate can present with incessant tachycardia but can be in a slower range of 180-200 bpms. The ECG changes can be subtle with normal $\mathrm{p}$ waves ( $\mathrm{P}$ wave axis could be abnormal) but the persistent tachycardia, disproportionately long PR interval for the tachycardia and impairment of ventricular function could be useful pointers towards this diagnosis. EAT could lead to impairment of ventricular function if poorly controlled. Medications to control the tachycardia can help with eventual recovery of ventricular function as this case has highlighted.

\section{NEONATAL READMISSIONS TO THE PAEDIATRIC WARDS DURING THE FIRST WAVE OF THE PANDEMIC: 3 CENTRE STUDY IN NORTHERN IRELAND}

Katarina Stefkova, Andrea Stobo, Laura Johnston, David Grier, Chloe Lynas. UK

\subsection{6/bmjpo-2021-RCPCH.142}

Background Readmissions of infants younger than 28 days back into hospital are highly undesirable. It is upsetting for the families and puts babies at risk of hospital acquired 